\title{
ON THE IFEL EXPERIMENT AT THE UCLA NEPTUNE LAB*
}

\author{
P. Musumeci ${ }^{\mathrm{a}}$, C. Pellegrini ${ }^{\mathrm{a}}$, J. B. Rosenzweig ${ }^{\mathrm{a}}$, A. Varfolomeev ${ }^{\mathrm{b}}$, S. Tolmachev ${ }^{\mathrm{b}}$, T. Yarovoi ${ }^{\mathrm{b}}$ \\ ${ }^{a}$ Department of Physics, University of California at Los Angeles, 405 Hilgard Ave, Los Angeles, CA \\ 90095, USA \\ ${ }^{\mathrm{b}}$ Coherent Radiation Laboratory, RRC "Kurchatov Institute", Moscow 123182, Russia
}

\begin{abstract}
We present an Inverse Free Electron Laser accelerator proposed for construction at the UCLA Neptune Lab. This experiment will use a $1 \mathrm{TW} \mathrm{CO} 2$ laser to accelerate through two strongly tapered undulators an electron beam from $16 \mathrm{MeV}$ up to $55 \mathrm{MeV}$. The scheme proposed is the diffraction dominated IFEL interaction. The Raleigh range of the laser beam is about $2 \mathrm{~cm}$, much shorter than the interaction length (the undulator length is $50 \mathrm{~cm}$ ). In this regime adiabatic capture is possible in the first part of the undulator. In the focus region, we propose a solution to the problem of the dephasing between electrons and photons due to the Guoy phase shift. Ponderomotive effects and implications for tolerancies are also studied.
\end{abstract}

\section{INTRODUCTION}

Inverse Free Electron laser schemes to accelerate particles have been proposed as advanced accelerators since many years [1-3]. Particular interest in this acceleration scheme comes from the unique characteristics of the output beam of this accelerator. Proof-of-principle IFEL experiments [4-5], including the staging of two different IFEL sections [6] have been carried out successfully. A lot of interest is currently in the control of the longitudinal phase space beam structure: femtosecond microbunches and few percent energy spread coming from the particle trapping in the accelerating bucket are theoretically possible. The purpose of the UCLA experiment is to achieve a substantial energy gain and investigate the longitudinal structure of the electron beam.

At the Neptune Laboratory at UCLA[7] there is the unique opportunity of having a high power laser and a relativistic electron beam in the same experimental facility. In the scheme, the electron beam is coming from a photoinjector+booster linac system with energy of 14.0 $\mathrm{MeV}$, and the high power $\mathrm{CO} 2$ laser is focused by a lens (f/18) with focal distance of $2.6 \mathrm{~m}$ to tight spot of few hundreds microns. Because the Raleigh range is much shorter than the undulator length, as indicated elsewhere [8], the interaction is diffraction dominated.

*Work supported by U.S. Dept. of Energy grant DEFG03-92ER40693.
Another important point in this configuration is that a tight laser waist size requires strict tolerances for matching the electron trajectory and accelerating phase in the following undulator region. The other fundamental ingredient for the Inverse Free Electron Laser is the undulator that provides the coupling between photons and electrons. Strong tapering of both period and magnetic field amplitude is needed for high-gradient acceleration and there are strict requirements on the magnetic field in order to maintain phase synchronicity and preserve the accelerating bucket along the accelerator, especially around the high laser field focal region. The undulator magnet will be built in collaboration with Kurchatov Institute.

\section{IFEL CONFIGURATION}

\subsection{MARS laser}

Table 1: Laser parameters

\begin{tabular}{|l|c|}
\hline Laser Power & $400 \mathrm{GW}$ \\
\hline Laser Wavelength & $10.6 \mu$ \\
\hline Laser waist size $\left(\mathrm{w}_{0}\right)$ & $350 \mu$ \\
\hline Raleigh range & $3.6 \mathrm{~cm}$ \\
\hline
\end{tabular}

The optics configuration at the Neptune Laboratory for the IFEL accelerator is designed to focus $400 \mathrm{GW}$ of a $10.6 \mu \mathrm{CO}_{2}$ laser to a waist of $350 \mu$. Light diffraction effects are a critical point in this situation. The model adequate to describe the photons, is not anymore a quasi monochromatic plane wave, but rather the gaussian beam approximation. The electromagnetic fields driving the interaction are not constant along the undulator but increase to a peak at the focus and then decrease back down. Special care in the undulator magnetic field profile design has to be taken to preserve the synchronicity with the electrons after the Guoy phase shift that takes place in a small region around the focus. Even when this problem is solved with careful undulator magnetic field shaping, other effects related to the small laser beam size appear. The first study on the IFEL[9] showed that spatial jitter intrinsic in the optical $f / 18$ configuration together with the ponderomotive scattering effects could seriously degrade the electron acceleration. The strong laser electric fields at the focus are predicted to kick transversely the electrons. In order to stably match the electron trajectories and the 
synchronous phase with the photons in this critical region the laser beam size was increased to $350 \mu$. Because of damage threshold limitation on the last optical element in the $\mathrm{CO}_{2}$ beam line, this can be done only at the expense of reducing the power from the available $1 \mathrm{TW}$ to $400 \mathrm{GW}$. The electromagnetic fields at the focus are smaller and the transverse kick problem is less critical. Peak accelerating gradient is lower, but the high field region ( Raleigh range) is longer and substantial energy gain can still be achieved.

\subsection{Undulator}

Table 2: Undulator parameters

\begin{tabular}{|l|c|c|}
\hline & Initial & Final \\
\hline Undulator period & $1.5 \mathrm{~cm}$ & $5.0 \mathrm{~cm}$ \\
\hline $\begin{array}{l}\text { Magnetic field } \\
\text { amplitude }\end{array}$ & $0.12 \mathrm{~T}$ & $0.60 \mathrm{~T}$ \\
\hline K parameter & 0.2 & 2.8 \\
\hline Gap & $12 \mathrm{~mm}$ & $12 \mathrm{~mm}$ \\
\hline
\end{tabular}

In collaboration with Kurchatov Institute we are designing a tapered undulator to provide the necessary synchronism between electrons and photons[10].

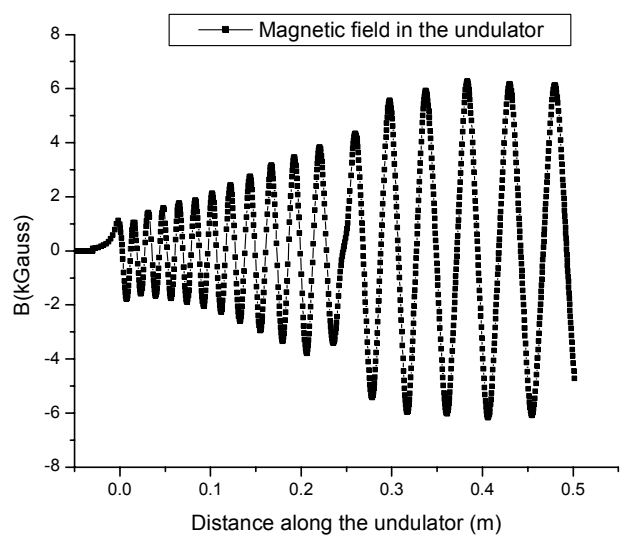

Fig.1: Magnetic field inside the undulator

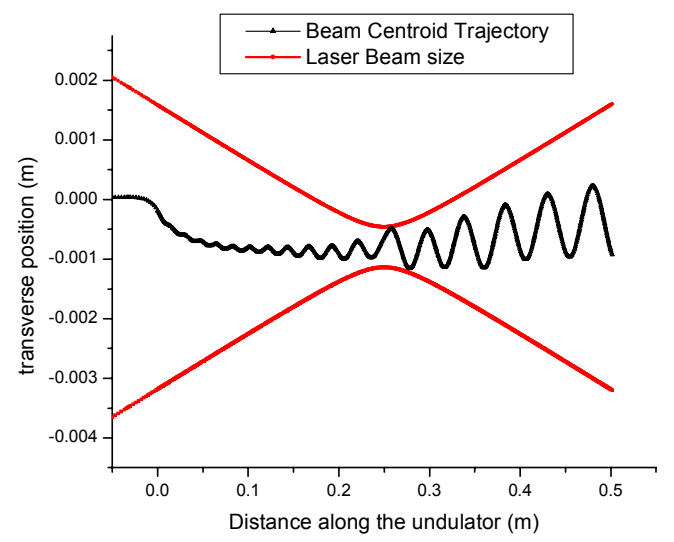

Fig.2: Electron trajectory. Laser beam envelope is also shown
The preliminary design is a hybrid permanent magnet planar undulator tapered in both period and magnetic field amplitude. Tapering of the magnetic field amplitude only, requires the field amplitude to increase to levels which are too large, and period tapering only makes soon the undulator too long for the space available in the experimental area. Magnetic fields are specially designed around the focus region to preserve synchronism with the laser light. Undulator parameters are given in Table 2. The undulator field simulations are done with the 3dimensional magnetostatic code RADIA[11].

\subsection{Electron beam}

To minimize the effects of ponderomotive scattering and more generally to sample a uniform region in the radiation fields, the electron beam should be focused to a spot size smaller than the laser beam size. This is possible because the transverse geometrical emittance of the electron beam is smaller than the one of the laser beam. The focusing properties of the undulator are negligible because the undulator-induced $\beta$-function is long. With a triplet upstream of the interaction region, we can focus the beam down to a spot of $150 \mu$. It is important that the initial electron beam energy is bigger than $14.0 \mathrm{MeV}$ to satisfy the synchronicity condition at the beginning of the undulator and optimize the trapping.

Table 3: Initial electron beam parameters

\begin{tabular}{|l|c|}
\hline Energy & $14.0 \mathrm{MeV}$ \\
\hline Energy spread (rms) & $0.5 \%$ \\
\hline Charge & $300 \mathrm{pC}$ \\
\hline Pulse length (rms) & $3 \mathrm{ps}$ \\
\hline Rms transverse emittance & $10 \mu$ \\
\hline Beam size at the focus & $150 \mu \mathrm{m}$ \\
\hline
\end{tabular}

\section{BEAM DYNAMICS}

Analysis of the IFEL performance is performed with the code TREDI[12]. The full Lorentz equations are solved. It should be noted that the strong period tapering and violent acceleration bring the approximations used to write the FEL equations (averaging over one undulator period) for the 1-dimensional IFEL theory to the limit of validity.

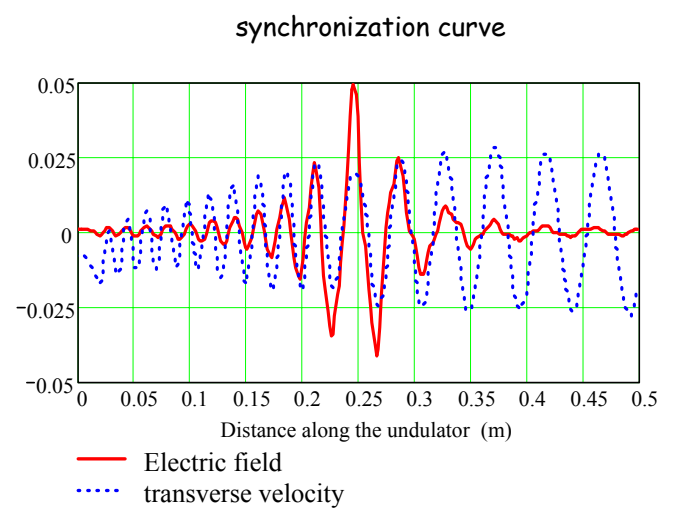

Fig3: Synchronization curve for the electrons. 
The particles are pushed through the analytical electromagnetic field of a gaussian mode representing the laser beam [13], and through the 3-dimensional magnetic field map from the code RADIA. Fig.3 shows the synchronization curve for the accelerated electrons. Looking at the output longitudinal phase space (fig.4), we see that particles trapping in the accelerating bucket took place. The beam quality at the output of the accelerator depends critically on the properties of the accelerating bucket. Different tapering can be used to improve one or the other of the characteristics of the bucket. Energy spread and micro bunch width could be adjusted depending on the final application. The choice for tapering was to optimize the trapped particle fraction. The characteristics of the output beam from the accelerator are reported in Table 4.

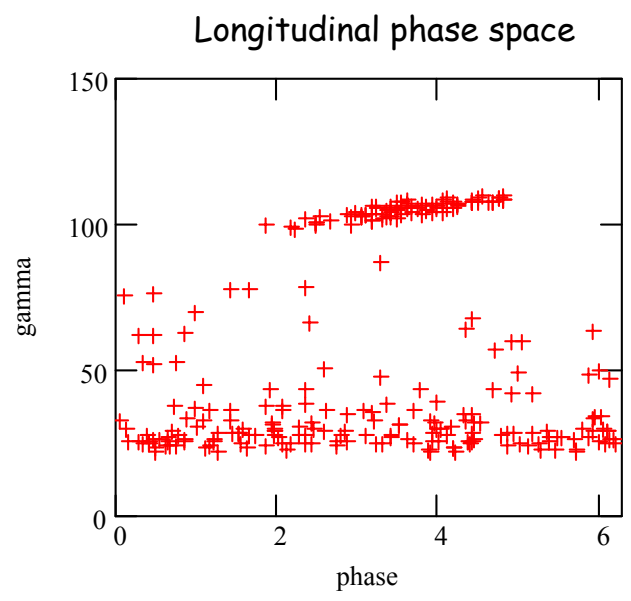

Fig.4: Longitudinal phase space of the electrons at the end of the IFEL accelerator.

Table 4: Output electron beam parameters

\begin{tabular}{|l|c|}
\hline Energy & $55 \mathrm{MeV}$ \\
\hline Energy spread (rms) & $2.5 \%$ \\
\hline Micro bunch length (rms) & $3 \mathrm{fs}$ \\
\hline Peak Current & $3 \mathrm{kA}$ \\
\hline Rms transverse emittance & $10 \mu$ \\
\hline Trapped particles \% & $25 \%$ \\
\hline
\end{tabular}

\section{STUDY OF ACCEPTANCES AND TOLERANCES}

In the study of the acceptances and tolerances of the accelerator, it was observed that changing the initial parameters does not affect the maximum final energy reached by the electrons. Because of the stability of the accelerating region in the phase space, for small variation of the parameters around the design, some particles will still be trapped and accelerated to the final energy. The most sensitive parameter is in this case the number of accelerated electrons. The tolerances refer to cases in which the fraction of captured particle is bigger than $20 \%$. Table 5 shows the results of this analysis.

Table 5: Tolerances of IFEL accelerator

\begin{tabular}{|l|c|}
\hline Energy & $14.0 \div 14.5 \mathrm{MeV}$ \\
\hline Laser Power & $350 \div 500 \mathrm{GW}$ \\
\hline Laser displacement & $-100 \div+100 \mu \mathrm{m}$ \\
\hline Laser angle misalignment & $-1 \div+1 \mathrm{mrad}$ \\
\hline
\end{tabular}

\section{CONCLUSIONS}

The goal of the IFEL project at the UCLA Neptune Laboratory is to achieve a substantial energy gain $(\sim 50$ $\mathrm{MeV}$ ), and to study the longitudinal phase space characteristics of the output beam. In this sense, it can be considered one of the first "second generation" advanced accelerator experiment where the main question is not if the scheme will work in principle, but what can the accelerator deliver in terms of beam quality and numbers of electrons per bunch. Future upgrades of the project include the possibility of using the full power that MARS laser is capable of (1 TW), either upgrading the last mirror on the $\mathrm{CO} 2$ optical beam line to overcome the damage threshold limitations, or including a laser beam splitter in order to split the available power and stage two different IFEL sections.

\section{REFERENCES}

[1]R.Palmer, J.Appl.Phys. 43, 3014, 1972

[2]E. Courant, C.Pellegrini, W.Zakowicz, Phys.Rev A $32,2813,1985$

[3]A.Fisher, J.Gallardo, J.Sandweiss, A.Van Steenbergen, Proc.Adv.Accel.Concpets, Port Jefferson, NY, AIP 279, p.299, 1993

[4]I.Wernick and T.C. Marshall, Phys Rev. A 46, 3566, 1992

[5]A.Van Steenbergen, J.Gallardo J.Sandweiss, J.Fang, M.Babzien, X.Qiu, J.Skaritka, X-J. Wang, Phys Rev Let. 77, 2690,1996

[6]W. Kimura, these proceedings, or to be published in Proc. Adv. Accel Concepts, Santa Fe NM, 2001

[7]C.Clayton, C.Joshi, K.Marsh, C.Pellegrini, J.Rosenzwig, PAC 97 Proc., 678, 1997

[8] P.Musumeci, C. Pellegrini, in Proc. Adv. Accel. Concepts, Santa Fe, NM, 2001

[9] A.A. Varfolomeev, S.V. Tolmachev, T.V. Yarovoi, RRCKI, Int. report CRL 02-01, 2001

[10] A.A. Varfolomeev, S.V. Tolmachev, T.V. Yarovoi, RRCKI, Int. report CRL 03-01, 2001

[11]P. Elleaume, et al. PAC 97 Proc., 3509, 1997

[12]F.Ciocci, L.Giannessi, A.Marranca, M.Quattromini, L.Mezi, Nucl.Instr.Met. 393, 434, 1997

[13]A. Siegman, University Science Books, 1986 